\title{
Reflexión sobre el desarrollo de competencias de los estudiantes a través de una actividad fuera del aula
}

\author{
Carlos JimÉnEZ NARros \\ Universidad Nebrija \\ cjimenez@nebrija.es \\ Fernando Toledano Cuervas-Mons \\ Universidad Nebrija \\ ftoledan@nebrija.es
}

Recibido: $23 / 10 / 2012$

Aceptado: 23/01/2013

\section{Resumen}

En este artículo se presenta una reflexión sobre el Concurso Gráfica Nebrija, una actividad extra-académica que cuenta con más de diez años de experiencia. Para cada edición, se forman equipos de trabajo de estudiantes de Publicidad y profesores, con el fin de que los profesores ayuden a los estudiantes a adquirir objetivos formativos específicos y, a la vez, supervisen la actividad de los alumnos recreando un ambiente de trabajo profesional. Con la implantación del EEES, las dinámicas y las metodologías de trabajo de ediciones anteriores se adaptaron para favorecer la adquisición de las competencias descritas en Bolonia que permiten a los estudiantes afrontar satisfactoriamente su carrera profesional. De esta manera, se ha adoptado un enfoque interdisciplinar que ha permitido incorporar a estudiantes de las titulaciones de periodismo y comunicación audiovisual.

Palabras clave: actividad formativa, educación universitaria, estudiantes de comunicación, actividad práctica, experiencia.

\section{Reflections upon the development of student's competences through an extra curricula activity}

\begin{abstract}
This article presents a reflection upon the use of extra-curricular activities, precisely the Print Nebrija Contest, now on its $10^{\text {th }}$ edition. Each edition counts on working teams of advertising students and lecturers, being lecturers' task to supervise the acquisition of objectives and help students to carry out the activity in a real professional working context. With the EEES, the teaching methodologies and group dynamics used in early editions had to be adapted in order to allow students to acquire the new competences and skills Bologna agreements specify and to allow students to face satisfactorily their professional career. In its first editions this Contest was organised by Advertising students but, since the EEES, an interdisciplinary approach has been adopted and students from the field of Journalism and Audiovisual Communication have been incorporated.

Keywords: formative action, tertiary education, Communications students, student's practice activity, experience.

\section{Referencia normalizada}

JIMÉNEZ NARROS, Carlos y TOLEDANO CUERVAS-MONS, Fernando (2013): "Reflexión sobre el desarrollo de competencias de los estudiantes a través de una actividad fuera del aula". Estudios sobre el Mensaje Periodístico. Vol. 19, Núm. especial marzo, págs.: 263-270. Madrid, Servicio de Publicaciones de la Universidad Complutense.
\end{abstract}

Sumario: 1. Introducción. 2. Contexto. 3. Metodología. 4. Resultados. 5. Conclusiones. 6. Referencias bibliográficas 


\section{Introducción}

Con la llegada del Espacio Europeo de Educación Superior (EEES) la universidad española ha experimentado una transformación en todos los aspectos, incluso en la docencia, ya que la innovación e investigación, unida a la aparición de las nuevas tecnologías, está posibilitando unas mejoras en este campo. Asistimos a un auge de nuevas fórmulas y formas docentes que posibilitan innumerables recursos al profesorado. (Cuadrado y Fernández Antelo, 2008: 202 y 204) Nuevas herramientas asíncronas que son las que permiten una comunicación en diferido entre usuarios, como pueden ser: foros de discusión, weblogs, FAQs, wikis y otras. Y las herramientas síncronas que facilitan la comunicación en directo entre usuarios, como pueden ser: chats y videoconferencia, entre otras.

El papel del estudiante también se ha modificado, ahora es el protagonista de su educación y ya no ejerce un rol pasivo, la interactividad significativa (Alemañy, 2009) en la que los alumnos deben construir su propio conocimiento, en las que el docente debe diseñar estrategias orientadas a "aprender a aprender" y "aprender a hacer". Esta transformación del alumno se podría comparar con las nuevas formas comunicativas (en especial publicitarias, la llamada Nueva Publicidad) en las que el receptor, gracias a la utilización de las nuevas tecnologías, se convierte en emisor, en lo relativo a generar nuevos contenidos (para la marca), algo muy cercano a la denominada narrativa transmedia.

Una de las fórmulas docentes o filosofías formativas que han supuesto una verdadera aproximación al llamado espíritu Bolonia, son aquellas en las que se apuesta por la práctica o la acción como la forma más eficiente de enseñanza y su vinculación a la experiencia (Carballo, 2006).

\section{Contexto}

El departamento de Publicidad y Marketing de la Universidad Nebrija ha celebrado en mayo de 2012 la décimo tercera edición del Concurso Gráfica Nebrija, una experiencia formativa en la que los estudiantes del grado en Publicidad exhiben sus mejores trabajos creativos. La organización de esta actividad docente es anterior a la implantación del Espacio Europeo de Educación Superior (EEES), pero responde a ese mismo espíritu integrador de la enseñanza, en la que diferentes disciplinas se interrelacionan para la consecución de un objetivo común. Como también, en estas últimas ediciones la organización de la actividad, enfocada a alumnos de la titulación de Publicidad, se ha extendido a estudiantes del grado en Periodismo y de Comunicación audiovisual. De esta manera con la participación en la organización y con la realización de los trabajos presentados al concurso se ponen en práctica una serie de competencias y habilidades que son imprescindibles para el crecimiento profesional de cualquier comunicador.

El Concurso Gráfica Nebrija se desarrolla en dos jornadas. En la primera los estudiantes presentan las piezas a concurso y montan una exposición muy vistosa en el Campus de la Berzosa (Hoyo de Manzanares), sede de la Facultad de Ciencias de la Comunicación. Un jurado profesional formado por prestigiosos directores de arte y redactores de las principales agencias del país, analiza y valora sus trabajos. El recono- 
cimiento a los mejores anuncios de gráfica se desvela en la segunda jornada, que se hace coincidir con la edición anual del Festival Jóvenes Tocados por la Publicidad.

La consolidación de esta actividad ha permitido que en las últimas ediciones, algunos de los miembros del jurado profesional sean antiguos alumnos de la Nebrija y que en su etapa de estudiantes también fueron premiados. Un paso más en la aproximación entre el mundo académico y el profesional.

El Concurso Gráfica Nebrija surgió en la primavera de 1999, como una actividad formativa complementaria y de apoyo a otra, el Festival Jóvenes Tocados por la $\mathrm{Pu}$ blicidad, encuentro muy reconocido en el sector de la publicidad. Este festival surgió dos años antes y está organizado íntegramente por la Facultad de Ciencias de la Comunicación. Su principal objetivo es premiar los mejores anuncios de cada año, emitidos en televisión y campañas digitales, visionadas por Internet. Los alumnos votan las campañas a través de distintas fases de investigación (recuerdo espontáneo, recuerdo sugerido y visionado) reforzados además con la realización de varios focus group formados por jóvenes universitarios. Para la puesta en marcha del festival se establece un organigrama de trabajo muy semejante al que puede encontrarse en una agencia de publicidad. Los estudiantes según su perfil y afinidad eligen el departamento en el que desarrollarán sus tareas (patrocinio, investigación, creatividad, prensa, relaciones públicas y producción). Cada departamento está dirigido por un alumno y supervisado por un profesor de la titulación. El festival publicitario anual culmina con una entrega de premios a las agencias publicitarias ganadoras. En respuesta a esta experiencia formativa nace la primera edición del Concurso Gráfica Nebrija, en la que a la inversa que el Festival Jóvenes Tocados, los profesionales premian, en distintas categorías, las piezas de los estudiantes.

El concurso aparece como una acción táctica más, para generar notoriedad de la imagen de marca de la Universidad Nebrija y, sobre todo, con el fin de vincular el mundo profesional con el académico.

A comienzos de cada curso académico se hacen públicas las bases de participación del concurso y se especifican los criterios para poder participar. Cualquier alumno matriculado en los estudios de Publicidad puede presentar sus trabajos, siempre que reúna las características técnicas exigidas, tamaño de la pieza (formato A-4) y resolución de imagen. Cada anuncio va acompañado de su correspondiente ficha en la que se indica el nombre y apellidos del estudiante, grupo al que pertenece, título de la pieza y marca anunciadora. Además todas las piezas se entregan en soporte digital para facilitar su posterior edición en formato DVD.

Todos los tutores de la titulación informan de la actividad a sus respectivos alumnos y al claustro de profesores, con la intención de conseguir la máxima participación posible en cada una de las convocatorias.

El Concurso Gráfica Nebrija se concibe como una actividad de innovación docente en la que los estudiantes son el eje principal. Fue una iniciativa pionera en fomentar habilidades y competencias que hoy encarnan el auténtico espíritu de Bolonia.

Los alumnos de Publicidad presentan todas las piezas creativas que desean y sólo acceden al concurso las mejores, tras ser previamente seleccionadas por un comité de profesores del área de creatividad. El certamen consta de varias categorías de premio 
que han ido cambiando a lo largo del tiempo para conseguir que las piezas sean lo más completas posibles. En las primeras ediciones las categorías fueron: Gran Premio Gráfica Nebrija, Mejor titular, Mejor body copy, Mejor Slogan, Mejor Tratamiento Tipográfico, Mejor Ilustración y Mejor Layout. En la sexta edición se crean nuevas categorías: Primer Premio Gráfica Nebrija, Segundo Premio Gráfica Nebrija, Tercer Premio Gráfica Nebrija, manteniéndose la categoría de Mejor Tratamiento Tipográfico, remodelándose las categorías referidas al texto del anuncio -Mejor Trabajo Copy- y renombrándose las referidas al aspecto visual del anuncio -Mejor Imagen y Mejor Trabajo Arte-. Como curiosidad destacar la creación de premios singulares como el Premio Joven Promesa y el Premio Postalfree. El primero de ellos está destinado a fomentar el interés por la publicidad entre los estudiantes de bachillerato. El segundo tiene como requisitos el formato postal y trabajar con briefings reales proporcionados por marcas patrocinadoras del concurso (Médicos sin fronteras, World Vision, Los 40, Heinz, CEAR). Esta categoría de premio se ha mantenido hasta la décimo segunda edición.

En la novena edición desaparecen los premios de Mejor Tratamiento Tipográfico y Mejor Imagen y las categorías Mejor Trabajo Copy y Mejor Trabajo Arte pasan a llamarse Mejor Redacción Publicitaria y Mejor Dirección de Arte. Finalmente en la décimo primera edición, para evitar que los alumnos orienten sus trabajos a la particularidad de cada premio, se apuesta por otras valoraciones. Se persigue que las piezas presentadas reúnan los mejores aspectos comunicativos, creativos y estéticos. En este sentido los galardones se simplificaron y ordenaron de la siguiente manera: Primer, Segundo y Tercer Premio Gráfica Nebrija y Mención Especial del Jurado. En esta última categoría se valora, bien la calidad del conjunto de anuncios presentados por un mismo estudiante, o bien aquella pieza cuyo arte final podría verse impresa en cualquier medio gráfico.

Las piezas presentadas a concurso - en muchas ocasiones- son resultado de las actividades académicas realizadas en las sesiones docentes de diferentes asignaturas del área de creación: Elementos del diseño gráfico, Herramientas del diseño publicitario, Creatividad publicitaria y Dirección de arte.

El montaje de la exposición también lo diseñan un pequeño grupo de alumnos que forman parte de la organización del concurso, siempre asesorado por profesores. Esta actividad responde al aprendizaje cooperativo (Benito y Cruz, 2005: 16) en el que de manera conjunta profesores y alumnos generan conocimiento siempre en un contexto de motivación personal, responsabilidad compartida y toma de decisiones.

En cada edición se elige una idea, entre varias propuestas, que determina el resultado visual de la exposición. El concepto seleccionado se utiliza en todos los materiales creativos. A modo de ejemplo, podríamos destacar dos ediciones. En la quinta, el concepto de comunicación fue "ideas listas para colgar", en que la exposición mostraba un tendal con los anuncios colgados con pinzas de la ropa, las categorías estaban impresas sobre camisetas y la serigrafía del DVD era la puerta de una lavadora. En la sexta edición, el estudiante Hugo Espinoza creó el concepto "dangertising" resultado de unir las palabras inglesas (danger - peligro y advertising - publicidad) y sobre este concepto de comunicación se basaron los distintos materiales utilizados para la ex- 
posición: una malla metálica para sujetar los anuncios, los carteles de las categorías simulaban señales de advertencia.

Un jurado profesional, elegido entre los mejores directores de arte y redactores de reconocidas agencias, juzga los anuncios que han sido expuestos finalmente. Tapsa, Contrapunto, Shackleton, Código Visual, Zapping, Señora Rhusmore, Remo, Mc Cann Erickson, Bob, El Laboratorio, Saatchi \& Saatchi, Kitchen, Young \& Rubicam, JWT, Publicis, Yslandia, SCPF, TBWA, Dommo, y DDB son algunas de las agencias que han colaborado con nosotros en lo largo de todas las ediciones.

La exposición, primero se monta en el Campus de la Berzosa, donde se convoca al jurado, formado por cinco o siete creativos que son quienes realizan una primera lista corta con diez anuncios. Tras un debate enriquecedor y varias fases deliberatorias se designan los anuncios ganadores. La exposición permanece abierta durante varios días para que pueda ser visitada por toda la comunidad universitaria.

Con posterioridad, se traslada al Palacio de Congresos y Exposiciones de Madrid para hacerla coincidir con el Festival Jóvenes Tocados por la Publicidad. El vestíbulo, espacio donde se instala, convierte la exposición en un lugar de encuentro y relación entre profesionales y estudiantes. De esta manera se consigue que los alumnos ganadores sean protagonistas y que sus trabajos puedan ser vistos por todos los profesionales que asisten a la gala. En muchas ocasiones este contexto ha sido el detonante para que distintos directores creativos se interesen por los trabajos y les ofrezcan prácticas en sus respectivas agencias.

La gala del festival comienza con la entrega de premios del Concurso de Gráfica Nebrija. Los miembros del jurado son quienes entregan el galardón a los alumnos. Además de un diploma acreditativo de la Universidad, los estudiantes ganadores reciben, de mano de los redactores y directores de arte, diferentes regalos proporcionados por las marcas patrocinadoras.

\section{Metodología}

Se ha destacado que esta actividad formativa se creó con anterioridad al EEES, por lo que una vez implantados los grados de las titulaciones de comunicación en la Universidad Nebrija (curso académico 2008-2009) se ha ido adaptando el concurso para asegurar el cumplimiento de competencias y habilidades por parte de los estudiantes participantes.

La metodología de trabajo consiste en crear un equipo de trabajo específico para la organización y gestión de esta actividad, el cual está formado por alumnos de $\mathrm{Pu}$ blicidad aunque, a partir del Plan de Bolonia, se ha ampliado a estudiantes de Periodismo y de Comunicación audiovisual. Se nombran dos directores de cuarto o tercer curso de sus titulaciones, normalmente es un alumno de Publicidad y otro de Periodismo o de comunicación audiovisual, que son los encargados de gestionar, coordinar y organizar la actividad. Los directores reportan a dos profesores, uno del área de $\mathrm{Pu}$ blicidad y Marketing y el otro de Periodismo, que supervisan y ayudan a los alumnos en la puesta en marcha del concurso. El resto del equipo lo forman alumnos de las tres titulaciones y de todos los cursos aunque, por lógica, el peso recae sobre los de tercero y cuarto pero es una magnífica experiencia de trabajo para los de primero y segundo. 
La forma de trabajar adquiere un cariz previo al mundo profesional, en el que no existe una calificación por en medio sino que la forma de evaluación se adhiere a la consecución de objetivos, los cuales están relacionados con las competencias y habilidades de las titulaciones.

El equipo de estudiantes encargados del concurso está en relación con los departamentos, formados también por alumnos, del Festival Jóvenes Tocados por la Publicidad ya que ambas actividades están estrechamente ligadas, como se ha explicado en el apartado anterior.

Dentro del equipo de trabajo específico del concurso se reparten distintas funciones a los alumnos participantes. La principal, tal y como hemos apuntado con anterioridad, consiste en la creación de un concepto creativo y la plasmación visual de cada edición del concurso, realización de carteles, montaje de la exposición y ejecución de acciones comunicativas con el fin de motivar a sus compañeros para que participen al concurso, presentando sus trabajos.

Para ello los estudiantes se reúnen para pensar el concepto, preparan sesiones de brainstorming con el fin de llegar a una idea a la que se le pueda sacar el máximo partido. Una vez seleccionada la idea final, se realizan los bocetos, tanto del cartel como de las acciones comunicativas que se ejecutarán en la universidad para motivar a participar en el concurso. Los estudiantes presentan su concepto creativo y bocetos a los profesores, quienes deben aprobar las propuestas o indicar los cambios que consideren.

El siguiente paso consiste en comunicar el Concurso Gráfica Nebrija a la comunidad universitaria, es un objetivo complicado dado el gran número de concursos que convocan a estudiantes. Los alumnos encargados de la puesta en marcha, bajo la supervisión de los profesores, realizan el cartel del evento y lo distribuyen por los distintos campus de la universidad junto con las bases del concurso; envían un nota de prensa a los boletines informativos internos; suben información a la página web $\mathrm{y}$, además, dinamizan el concurso a través de las redes sociales, desde el inicio hasta la finalización del mismo.

Al mismo tiempo, otros alumnos que forman este equipo de trabajo específico para la organización del concurso, escriben guiones para piezas audiovisuales que formarán parte de la campaña. Anuncios que grabarán y editarán, una vez hayan sido aprobado por los profesores.

Este trabajo profesor y estudiantes no se limita a la mera supervisión o aprobación de materiales sino que se crea una relación especial y eficaz. Se crean verdaderos equipos de trabajo en los que ambos profesores participan activamente y, sobre todo, motivan y ayudan a los alumnos. Hay que resaltar que a los alumnos de grado se les otorgan créditos por participar en este equipo de trabajo aunque su recompensa saben que no sólo es esa. La formación impartida en las aulas tiene una ayuda adicional y los estudiantes así lo perciben.

\section{Resultados}

La actividad formativa pone a prueba muchas de las competencias que necesitan conseguir los estudiantes del grado en Publicidad, en concreto en el área de creatividad. 
Aunque se ha intentado integrar a alumnos de Periodismo y Comunicación audiovisual. El concurso ya ha superado su décimo tercera edición, y se anticipó al EEES, momento en que se materializaron una a una las competencias genéricas y específicas de los títulos.

Como ya anticipamos, a lo largo de su itinerario formativo, las asignaturas más comprometidas con el concurso son: Elementos del diseño gráfico, Herramientas del diseño publicitario, Creatividad publicitaria y Dirección de arte. Todas estas materias las cursan durante los tres primeros años de su titulación. Las dos primeras las realizan en su primer ciclo y las dos últimas en tercer curso. Con estas asignaturas adquieren los conocimientos básicos sobre composición y sobre los diferentes elementos de diseño; formato, color, tipografía e imagen. De esta manera pueden elaborar documentos gráficos con sentido comunicativo, creativo y estético. Manejan distintas aplicaciones digitales (Illustrator y Photoshop) y se familiarizan con la tecnología. Desarrollan trabajos en equipo de forma eficaz, utilizan su ingenio y buscan soluciones creativas a problemas de comunicación concretos. Para su desarrollo profesional simulan los roles del redactor publicitario (copy) y director de arte. (Echevarría, 2002: 18) Existen cuatro tipos de capacidades. Competencia técnica (saber), poseer conocimientos especializados y relacionados con el ámbito profesional. Competencia metodológica (saber hacer) aplicar los conocimientos a situaciones laborales concretas. Competencia participativa (saber estar) demostrar un comportamiento orientado hacia el grupo. Competencia personal (saber ser), asumir responsabilidades y tomar decisiones.

Al igual que se amplia el abanico de perfiles, se extiende la variedad de competencias y habilidades. Relacionadas con la puesta en marcha del concurso, aparecen vínculos con otras asignaturas de la titulación de Publicidad como "Publicidad interactiva" y del área de las Relaciones Públicas, asignaturas como "Principios de las RR.PP.” " “Técnicas de las RR.PP.” Asimismo aparecen asignaturas propias del grado en Periodismo como "Técnicas narrativas", "Redacción periodística en los medios" y "Comunicación institucional". Y de la titulación de comunicación audiovisual: "guión en otros medios audiovisuales", "Dirección de Fotografía: Cámara e iluminación", "Dirección Escénica: Artística y de Actores" y "Edición y montaje".

El resultado de esta actividad a lo largo de las ediciones ha sido muy motivador. La participación de los estudiantes es muy destacable, el saber y el saber hacer se conjugan perfectamente y así convierten el concurso en un maravilloso escaparate de su trabajo. Muchas gracias a todos los alumnos que han participado en esta experiencia, pues desde el principio se volcaron con gran interés en la realización y puesta en marcha de esta actividad formativa.

\section{Conclusiones}

La trayectoria del Concurso Gráfica Nebrija es una actividad motivadora para los estudiantes que se ha podido amoldar al Espacio Europeo de Educación Superior (EEES) y que se caracteriza por unos determinados valores:

1. Permite a los alumnos desarrollar algunas de las competencias de sus titulaciones bajo un prisma de motivación y compromiso. Es un refuerzo para la formación te- 
órica y práctica de la titulación de Publicidad aunque en las últimas ediciones se ha extendido al grado de Periodismo y de Comunicación audiovisual.

2. Crea un escenario real en el que los estudiantes se agrupan en función de sus habilidades e intereses, en el que no importa el curso ni la titulación en la que estudian sino lo que puedan aportar al equipo.

3. Simula una situación profesional en la que tanto los estudiantes como los profesores, involucrados en la organización del concurso, trabajan en la consecución de objetivos y resultados concretos con lo que se crea una relación más cercana a compañeros de trabajo.

4. Configura un escaparate del trabajo de los estudiantes que sirve para crear un vínculo entre el mundo docente y el mundo profesional.

\section{Referencias bibliográficas}

ALEMAÑY, Cristina (2009): "Un nuevo rol docente en la era de las nuevas tecnologías. Cuadernos de Educación y desarrollo". Cuadernos de Educación y Desarrollo, vol. 1, No. 1, Marzo. Universidad de Málaga.

BENITO, Águeda y CRUZ, Ana (2005): Nuevas claves para la docencia Universitaria en el Espacio Europeo de Educación Superior. Madrid, Narcea Ediciones.

CARBALLO, Roberto (2006): “Aprender haciendo. Guía para profesores: Aproximación a los espacios de Aprendizaje basados en la acción, la experiencia y el grupo de trabajo y aplicaciones prácticas". II Encuentro sobre experiencias grupales innovadoras en la docencia universitaria. Centro de Estudios Superiores Felipe II. Universidad Complutense.

CUADRADO, Isabel y FERNÁNDEZ ANTELO, Inmaculada (2008): "Nuevas competencias del profesor en el EEES: una experiencia de innovación docente". Revista Electrónica Teoría de la Educación. Educación y Cultura en la Sociedad de la Información (Vol. 9, $\mathrm{n}^{\circ}$ 1). Universidad de Salamanca.

ECHEVERRÍA, Benito (2002): Gestión de la competencia de acción profesional. Revista de Investigación Educativa, Volumen 20, no 1.

\section{Carlos JIMÉNEZ NARROS}

Universidad Nebrija

Coordinador académico Grado en Periodismo

cjimenez@nebrija.es

\section{Fernando TOLEDANO CUERVAS-MONS}

Universidad Nebrija

Coordinador académico Grado en Publicidad

ftoledan@nebrija.es 